\title{
Analyticity Constraints for Hadron Amplitudes: Going High to Heal Low Energy Issues
}

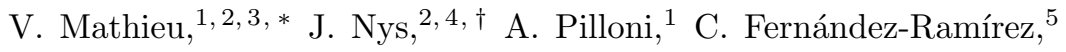 \\ A. Jackura, ${ }^{2,3}$ M. Mikhasenko, ${ }^{6}$ V. Pauk, ${ }^{1}$ A. P. Szczepaniak,,${ }^{1,2,3}$ and G. Fox ${ }^{7}$ \\ (Joint Physics Analysis Center) \\ ${ }^{1}$ Theory Center, Thomas Jefferson National Accelerator Facility, Newport News, VA 23606, USA \\ ${ }^{2}$ Center for Exploration of Energy and Matter, Indiana University, Bloomington, IN 47403, USA \\ ${ }^{3}$ Physics Department, Indiana University, Bloomington, IN 47405, USA \\ ${ }^{4}$ Department of Physics and Astronomy, Ghent University, Belgium \\ ${ }^{5}$ Instituto de Ciencias Nucleares, Universidad Nacional Autónoma de México, Ciudad de México 04510, Mexico \\ ${ }^{6}$ Universität Bonn, Helmholtz-Institut für Strahlen- und Kernphysik, 53115 Bonn, Germany \\ ${ }^{7}$ School of Informatics, Computing, and Engineering, \\ Indiana University, Bloomington, IN 47405, USA
}

\begin{abstract}
Analyticity constitutes a rigid constraint on hadron scattering amplitudes. This property is used to relate models in different energy regimes. Using meson photoproduction as a benchmark, we show how to test contemporary low energy models directly against high energy data. This method pinpoints deficiencies of the models and treads a path to further improvement. The implementation of this technique enables one to produce more stable and reliable partial waves for future use in hadron spectroscopy and new physics searches.
\end{abstract}

Introduction.- Determination of various hadronic effects represents a major challenge in searches for New Physics through precision measurements [1 [5]. For example, the possible identification of Beyond Standard Model signals in $B$ meson decays is hindered by uncertainties in hadronic final state interactions. The strongly coupled nature of QCD prevents us from computing these effects directly from the underlying microscopic formulation. Nevertheless, one can use the first principles of $S$ matrix theory to impose stringent constraints on hadron scattering amplitudes [6] 8 . These approaches are encountering a renewed interest even in the more formal context of strongly coupled theories 9 -11.

In this Letter, we show how to use analyticity to relate the amplitudes at high energies to the physics at low energies, where resonance effects dominate. This is not only important for reducing hadronic uncertainties in the aforementioned processes, but is of interest on its own merits for unraveling the spectrum of QCD. According to phenomenological predictions and lattice QCD simulations, the current spectrum summarized in the Particle Data Group (PDG) is far from complete [12]. For example, the recent discoveries of unexpected peaks in data indicate that the true hadron spectrum is far more complex than predicted 13 18. As a working case, we focus here on the baryon sector in the intermediate energy range. In the PDG these $N^{*}$ and $\Delta$ resonances are referred to as "poorly known" [12, despite the large amount of data available. The ambiguities encountered when identifying resonances are related to the fact that, as the center of mass energy increases, so does the number of contributing partial waves, vastly complicating the reaction models used in data analysis. The $2-3 \mathrm{GeV}$ mass region is of particular interest for baryon spectroscopy since, besides the ordinary quark model multiplets, it is expected to contain a new form of exotic light quark matter that is dominated by excitations of the gluon field [16, 19]. The recent upgrade at Jefferson Lab 20 24 is providing high statistics data on hadron photoproduction. New amplitude analysis methods are a prerequisite to achieve a robust extraction of hadron resonance parameters.

Many research groups carry out low energy, coupled channel, partial wave analyses (PWA) for baryon spectroscopy. Currently, the most active are ANL-Osaka [25], Bonn-Gatchina 26, 27, JPAC [28, 29], Jülich-Bonn [30, 31, MAID [32, and SAID [33, 34]. These groups perform global fits to hadro- and/or photoproduction data using a finite set of partial waves to extract baryon resonance properties 35,36 . In these approaches the high energy data are largely ignored. As we show in this Letter, these data can greatly impact the baryon spectrum analyses through analyticity. Specifically, we implement Finite Energy Sum Rules derived from dispersion relations [37, and use simple approximations to describe the high energy data. The sum rules relate the amplitudes in the baryon resonance region to the high energy dynamics, where the amplitudes are described by exchanges of meson Regge poles [38. We apply our method to the existing data on $\pi^{0}$ and $\eta$ photoproduction 39 41. These cases constitute a first step towards a straightforward and systematic implementation of high energy constraints into low energy amplitudes, and provide a template for further application in data analysis.

Analyticity constraints for photoproduction.- The reaction $\gamma p \rightarrow x p$, where $x=\pi^{0}, \eta$ is completely described in terms of four independent scalar amplitudes $A_{i}(s, t)$. These are analytic functions of the Mandelstam variables $s$ (the square of the center of mass energy) and $t$ (the square of the momentum transfer) [42. At fixed $t$, each $A_{i}(s, t)$ satisfies an unsubtracted dispersion rela- 
tion involving the discontinuity with respect to $s$ along the unitarity cut and the crossed-channel unitarity cut in $u=2 m_{p}^{2}+m_{x}^{2}-s-t$. Charge conjugation symmetry relates the discontinuity along the crossed channel cut to that of the direct channel. This symmetry is made explicit by writing the amplitude as a function of the variable ${ }^{1} \nu \equiv(s-u) / 2$. For large $|\nu|$ and small $t$ kinematics, the amplitudes are well approximated by Regge poles, i.e. via crossed channel exchanges. In this region, the amplitudes take the form

$$
\operatorname{Im} A_{i}(\nu, t)=\sum_{n} \beta_{i}^{(n)}(t) \nu^{\alpha^{(n)}(t)-1}
$$

The Regge poles are determined by the trajectories $\alpha^{(n)}(t)$ and the residues $\beta_{i}^{(n)}(t)$. The index $n$ runs over all possible exchanges. This approximation holds only if $|\nu|$ is greater than some cutoff $\Lambda$ above the resonance region. For $|\nu|<\Lambda$, the amplitude is dominated by direct channel resonances, and thus it can be well approximated by a finite number of partial waves. One can write a dispersion relation using Cauchy's theorem with the contour in Fig. 1. and calculate explicitly the integral in the circle $|\nu|=\Lambda$ assuming the form in Eq. (1). One readily obtains 38

$$
\int_{0}^{\Lambda} \operatorname{Im} A_{i}(\nu, t) \nu^{k} \mathrm{~d} \nu=\sum_{n} \beta_{i}^{(n)}(t) \frac{\Lambda^{\alpha^{(n)}(t)+k}}{\alpha^{(n)}(t)+k} .
$$

The amplitudes $A_{1,2,4}$ and $A_{3}$ are even and odd functions of $\nu$, respectively. Here $k$ is an arbitrary positive integer, odd for $A_{1,2,4}$ and even for $A_{3}$. We give the value of $\Lambda$ in terms of an energy cutoff $s_{\max }$, which introduces additional $t$ dependence $\Lambda=s_{\max }+\left(t-2 m_{p}^{2}-m_{x}^{2}\right) / 2$. We restrict the sum on the right hand side (rhs) of Eq. (2) to the dominant $t$-channel Regge poles. Each $A_{i}$ receives a contribution from both isoscalar and isovector exchanges. Natural parity exchanges (with $P=(-)^{J}$ ) dominate $A_{1}$ and $A_{4}$, while the unnatural ones (with $P=(-)^{J+1}$ ) dominate $A_{2}$ and $A_{3}$. More specifically, the $n=\rho, \omega$, Regge poles contribute to $A_{1}$ and $A_{4}$, while $A_{2}$ and $A_{3}$ are determined by exchanges of the $n=b, h, \rho_{2}, \omega_{2} \beth^{2}$

The trajectories are nearly degenerate for all the natural exchanges [45], and in the kinematical region of interest, they can be well approximated by

$$
\alpha(t) \equiv \alpha^{(\rho)}(t)=\alpha^{(\omega)}(t)=1+0.9\left(t-m_{\rho}^{2}\right),
$$

for $i=1,4$. For the unnatural exchanges, $\alpha(t)=0.7\left(t-m_{\pi}^{2}\right)$ for $i=2,3$. At high energy the contribution of unnatural versus natural exchanges to observables in the forward direction is suppressed. For

\footnotetext{
${ }^{1}$ As customary, all dimensional variables are given in units of $1 \mathrm{GeV}$.

${ }^{2}$ Even though there are some experimental indications of the existence of $\rho_{2}$ and $\omega_{2}$ 43, 44, they have been observed by one single group, and thus need further confirmation [12.
}

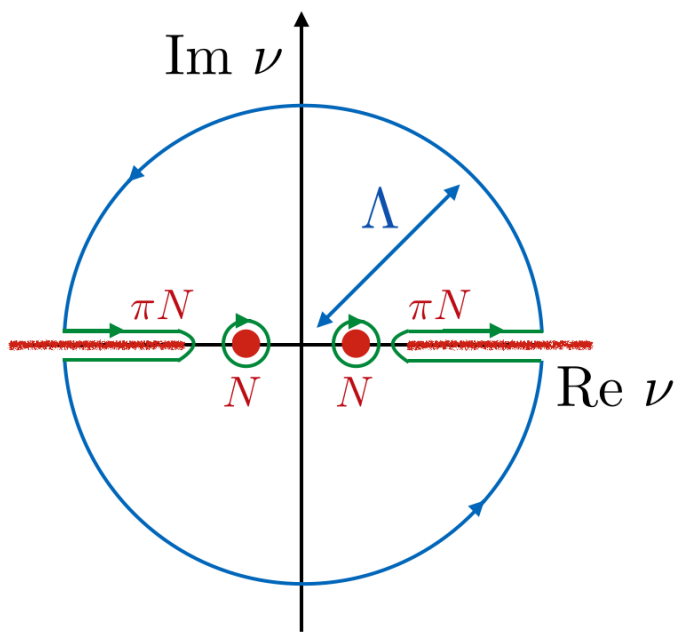

FIG. 1. Contour in the complex $\nu$-plane used in the derivation of the sum rules in Eq. 22. The radius $\Lambda$ must be taken sufficiently large, for the single Regge pole approximation to hold at $|\nu|=\Lambda$. The nucleon pole and the $\pi N$ cuts are shown on the real axis.

example, with a beam energy of $9 \mathrm{GeV}$, the suppression is expected to be $\nu^{2\left(0.9 m_{\rho}^{2}-0.7 m_{\pi}^{2}-1\right)} \sim 7 \%$. This can be compared with polarization observables, such as the beam asymmetry $\Sigma]^{3}$ which are sensitive to the interference between the natural and the unnatural Regge poles. If one neglects the unnatural contributions, $\Sigma=1$. The recent measurement of $\pi^{0}$ and $\eta$ beam asymmetries at GlueX [23] confirms that $\Sigma>0.9$, so that the unnatural exchanges contribute $\lesssim 5 \%$ to the observables. In the following, we will consider the amplitudes dominated by natural exchanges, $A_{1}$ and $A_{4}$, only. We use low energy models as input to determine the left hand side of Eq. (2), and use it to predict the residues. To this aim we define the effective residues,

$$
\widehat{\beta}_{i}(t)=\frac{\alpha(t)+k}{\Lambda^{\alpha(t)+k}} \int_{0}^{\Lambda} \operatorname{Im} A_{i}^{\mathrm{PWA}}(\nu, t) \nu^{k} \mathrm{~d} \nu,
$$

where $A_{i}^{\mathrm{PWA}}$ is the amplitude calculated from low-energy models.

Because of Regge trajectory degeneracy, the $\widehat{\beta}_{i}(t)$ 's describe the sum of the contribution of both isovector and isoscalar exchanges. Consistency of the single pole hypothesis requires the rhs of Eq. (4) to be independent of $\left.k\right|^{4}$ For $|\nu|>\Lambda$, the amplitudes can be expressed in

\footnotetext{
3 The beam asymmetry is $\Sigma \equiv\left(\mathrm{d} \sigma_{\perp}-\mathrm{d} \sigma_{\|}\right) /\left(\mathrm{d} \sigma_{\perp}+\mathrm{d} \sigma_{\|}\right)$, with $\mathrm{d} \sigma_{\perp(\|)}$ the differential cross section of the photon polarized perpendicular (parallel) to the reaction plane.

${ }^{4}$ For example, if one added another nondegenerate trajectory $\alpha_{2}<\alpha$, the effective residue would depend on $k$ as $\hat{\beta}_{i}=$ $\beta_{i}+\frac{\beta_{i, 2}}{\Lambda^{\alpha-\alpha_{2}}} \frac{\alpha+k}{\alpha_{2}+k}$. The latter becomes negligible for $\Lambda$ sufficiently large.
} 


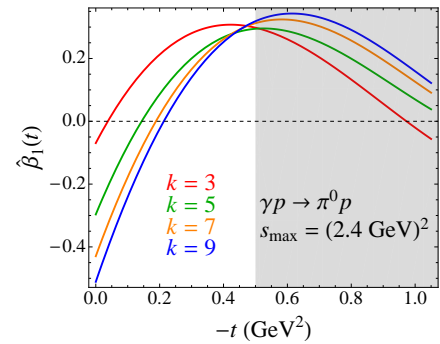

(a)

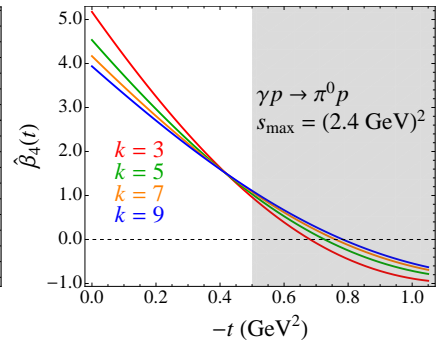

(b)

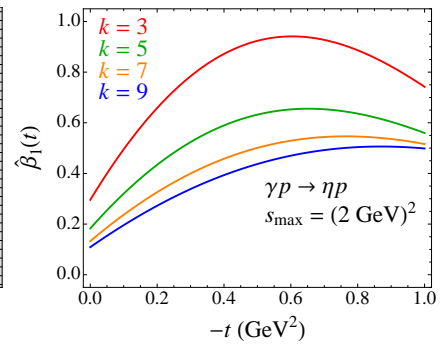

(c)

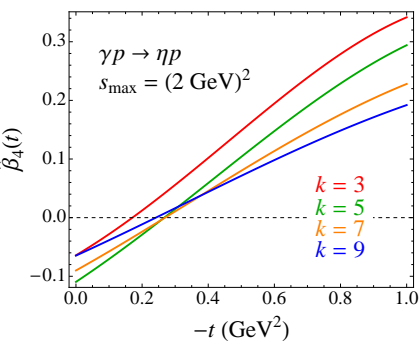

(d)

FIG. 2. Effective residues computed from the low energy models using Eq. (4). (a) and (b): $\pi^{0}$ photoproduction using SAID 33. (c) and (d): $\eta$ photoproduction using $\eta$-MAID 32 . For $\pi^{0}$, the single pole approximation is valid for $-t \lesssim 0.5 \mathrm{GeV}^{2}$, as explained in the text. The dispersion in $k$ is small for $\pi^{0}$, while the large variation with $k$ for $\eta$ indicates issues with the low energy model.

terms of the effective residues as 38

$$
\widehat{A}_{i}(\nu, t)=\left[i+\tan \frac{\pi}{2} \alpha(t)\right] \widehat{\beta}_{i}(t) \nu^{\alpha(t)-1} .
$$

The $\widehat{A}_{i}(\nu, t)$ are the high energy amplitudes calculated from the low energy models entering in the $\widehat{\beta}_{i}(t)$. Comparing the observables calculated with these to data allows us to check the quality of the low energy models. In the high energy limit, the differential cross section becomes

$$
\begin{aligned}
\frac{\mathrm{d} \widehat{\sigma}}{\mathrm{d} t} & \simeq \frac{1}{32 \pi}\left[\left|\widehat{A}_{1}\right|^{2}-t\left|\widehat{A}_{4}\right|^{2}\right] \\
& =\frac{\nu^{2 \alpha(t)-2}}{32 \pi}\left[1+\tan ^{2} \frac{\pi}{2} \alpha(t)\right]\left[\widehat{\beta}_{1}^{2}(t)-t \widehat{\beta}_{4}^{2}(t)\right] .
\end{aligned}
$$

Results. - We next discuss what these constraints can tell us about the existing low energy analyses. We consider $\widehat{\beta}_{i}(t)$ for $k=3,5,7,9$. For $\pi^{0}$, we use the SAID partial wave model which is valid up to $s_{\max }=$ $(2.4 \mathrm{GeV})^{2}$ [33. For $\eta$, the amplitudes need to be extrapolated below the physical $\eta N$ threshold, down to the $\pi N$ threshold (see Fig. 1). Among the various models, only $\eta$-MAID [32, valid only up to $s_{\max }=(2 \mathrm{GeV})^{2}$, is given in terms of analytical functions that allow for this continuation 46 .

The two effective residues $\widehat{\beta}_{1,4}(t)$ are shown in Figs. 2(a) and 2(b) for $\pi^{0}$ and in Figs. 2(c) and 2(d) for $\eta$, respectively. In the case of $\pi^{0}$, we restrict the analysis to the $0 \leq-t \leq 0.5 \mathrm{GeV}^{2}$ region, because of subleading Regge cut contributions which are known to dominate the cross section at higher $-t$ [47]. We note that the residues are fairly independent of $k$. Conversely, the dependence on $k$ for $\eta$ is large. This points to a problem in the low energy model. Possible reasons can be that the resonant content for energies less than $2 \mathrm{GeV}$ is underestimated, or the $2-3 \mathrm{GeV}$ resonances are relevant. In either case the low energy model can be improved using these constraints.

In Fig. 3(a) we predict the high energy $\pi^{0}$ differential cross section computed in Eq. (6) using the effective residues $\widehat{\beta}_{i}(t)$. Both the magnitude and shape of the $t$ dependence show a remarkable agreement with the data. The energy dependence is given by the trajectories in Eq. (3). In the region of interest, the $t$ dependence is fully determined by the low energy amplitudes through the integral over the imaginary part, see Eq. (2). There is a dip in the cross section data near $-t=0.5 \mathrm{GeV}^{2}$, which can be traced to the zero in the dominant $\widehat{\beta}_{4}(t)$ at $-t \simeq 0.7 \mathrm{GeV}^{2}$ in Fig. 2(b). The predictions are almost independent of the moment $k$. The $t$ dependence is identical for moments up to $k=9$, and the overall normalization changes by a maximum of $20 \%$.

The predictions for $\eta$ are shown in Fig. 3(b). Since the $\widehat{\beta}_{i}(t)$ computed from the low energy model have significant $k$ dependence, we show the cross section for fixed, $k=3$, which happens to have the correct overall normalization. The prediction agrees very well with data up to somewhat higher $-t$, but it underestimates the cross section in the forward, $-t<0.25 \mathrm{GeV}^{2}$ region. This effect originates from the small value of $\widehat{\beta}_{4}$ in this region, as can be seen on Figs. 2(c) and 2(d). It is worth noting that the available PWA models [25, 27, 31, 32] strongly disagree in this specific $t$ region. In particular, in $\eta$-MAID there is a peculiar cancellation between isoscalar and isovector exchanges, which results in a smaller effective residue [46]. This illustrates how the implementation of our approach can impact on the low energy analyses.

Conclusions. - We discussed a technique which uses analyticity to constrain low energy hadron effects with the high energy data. We have benchmarked it against meson photoproduction, one of the main reactions to study hadron spectroscopy. In this specific case, we showed the effectiveness of the approach in identifying potential deficiencies in the low energy models. We showed explicitly how the baryon spectrum determines the seemingly unrelated meson exchanges dominating forward scattering at high energies, and vice versa. Experiments at Jefferson Lab are currently exploring meson photoproduction above the baryon resonance region. The 


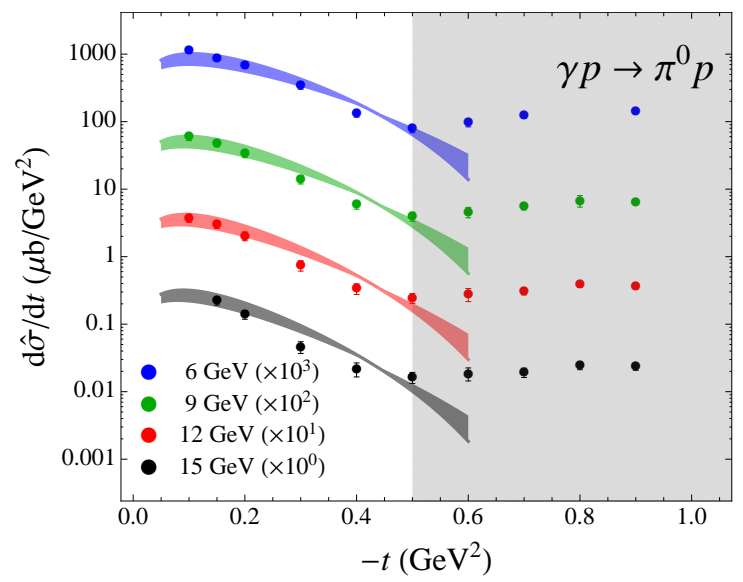

(a)

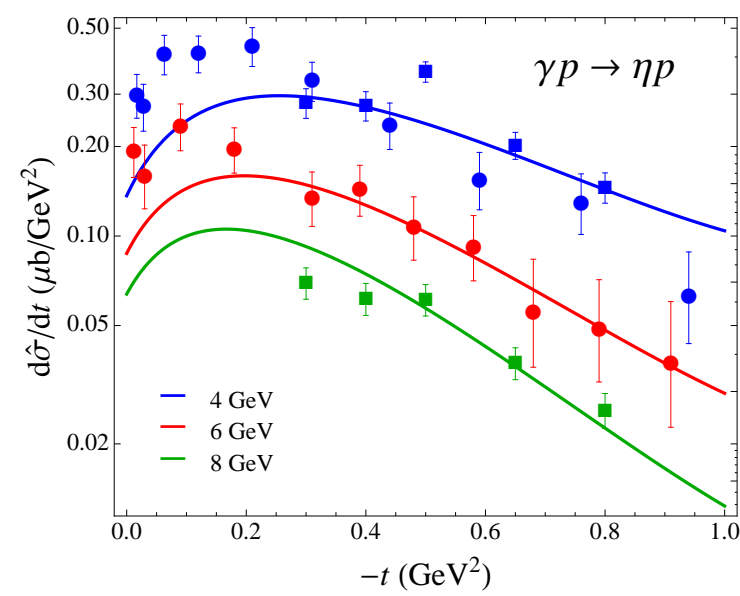

(b)

FIG. 3. Differential cross sections computed from the low energy models using Eq. (6). (a) $\pi^{0}$ photoproduction using SAID 33. The prediction is restricted to $-t<0.5 \mathrm{GeV}^{2}$, as explained in the text. The error band takes into account the (small) dispersion with $k$. The legend indicates the beam energy in the laboratory frame and the scaling factors. Data are from [40]. (b): $\eta$ photoproduction using $\eta$-MAID [32. Prediction is shown for $k=3$, as explained in the text. The legend indicates the beam energy in the laboratory frame. Data are from 39] (circles) and [41] (squares). For $\pi^{0}$ the prediction agrees with data, while for $\eta$ the depletion in the forward $-t<0.25 \mathrm{GeV}^{2}$ is a marker for an inconsistency of the low energy model.

technique presented here can be applied to these forthcoming data, and make a significant impact on baryon spectroscopy research. The approach can be extended to other hadron reactions, and help control the hadronic effects that drive the uncertainties in New Physics searches, especially in the heavy flavor sector.

Acknowledgments. - This material is based upon work supported in part by the U.S. Department of Energy, Office of Science, Office of Nuclear Physics under contract DE-AC05-06OR23177. This work was also supported in part by the U.S. Department of Energy under Grant DEFG0287ER40365, National Science Foundation under Grants PHY-1415459 and PHY-1205019, the IU Collaborative Research Grant, the Research Foundation Flanders (FWO-Flanders), PAPIIT-DGAPA (UNAM) grant No. IA101717, CONACYT (Mexico) grant No. 251817, and Red Temática CONACYT de Física en Altas Energías (Red FAE, Mexico).

* mathieuv@indiana.edu

† jannes.nys@ugent.be

[1] F. Archilli, M.-O. Bettler, and K. A. Petridis, Nature (London) 546, 221 (2017)

[2] G. Ciezarek, M. Franco Sevilla, B. Hamilton, R. Kowalewski, T. Kuhr, V. Lüth, and Y. Sato, Nature (London) 546, 227 (2017).

[3] A. Bevan et al. (Belle, BaBar), Eur.Phys.J. C74, 3026 (2014), arXiv:1406.6311 [hep-ex]

[4] F. Jegerlehner and A. Nyffeler, Phys.Rept. 477, 1 (2009) arXiv:0902.3360 [hep-ph]
[5] V. Cirigliano and M. J. Ramsey-Musolf, Prog.Part.Nucl.Phys. 71, 2 (2013), arXiv:1304.0017 [hep-ph]

[6] R. J. Eden, P. V. Landshoff, D. I. Olive, and J. C. Polkinghorne, The analytic S-matrix (Cambridge Univ. Press, Cambridge, 1966).

[7] G. F. Chew, S-Matrix Theory of Strong Interactions (W. A. Benjamin, Inc., New York, USA, 1961).

[8] G. F. Chew, Phys.Rev. D4, 2330 (1971).

[9] M. F. Paulos, J. Penedones, J. Toledo, B. C. van Rees, and P. Vieira, "The $S$-matrix Bootstrap I: QFT in AdS," (2016), arXiv:1607.06109 [hep-th].

[10] M. F. Paulos, J. Penedones, J. Toledo, B. C. van Rees, and P. Vieira, "The $S$-matrix Bootstrap II: Two Dimensional Amplitudes," (2016), arXiv:1607.06110 [hep-th].

[11] M. F. Paulos, J. Penedones, J. Toledo, B. C. van Rees, and P. Vieira, "The $S$-matrix Bootstrap III: Higher Dimensional Amplitudes," (2017), arXiv:1708.06765 [hepth]

[12] C. Patrignani et al. (Particle Data Group), Chin.Phys. C40, 100001 (2016).

[13] M. R. Pennington, J.Phys. G43, 054001 (2016), arXiv:1604.01441 [hep-ph]

[14] R. A. Briceño et al., Chin.Phys. C40, 042001 (2016), arXiv:1511.06779 [hep-ph]

[15] R. Aaij et al. (LHCb), Phys.Rev.Lett. 115, 072001 (2015), arXiv:1507.03414 [hep-ex]

[16] C. A. Meyer and E. S. Swanson, Prog.Part.Nucl.Phys. 82, 21 (2015), arXiv:1502.07276 [hep-ph]

[17] R. F. Lebed, R. E. Mitchell, and E. S. Swanson, Prog.Part.Nucl.Phys. 93, 143 (2017), arXiv:1610.04528 [hep-ph]

[18] A. Esposito, A. Pilloni, and A. D. Polosa, Phys.Rept. 668, 1 (2016), arXiv:1611.07920 [hep-ph].

[19] J. J. Dudek and R. G. Edwards, Phys.Rev. D85, 054016 (2012), arXiv:1201.2349 [hep-ph]. 
[20] M. Battaglieri et al. (CLAS), "JLab approved experiment E12-11-005: Meson Spectroscopy with low $Q^{2}$ electron scattering in CLAS12," (2011).

[21] M. Battaglieri et al. (CLAS), "JLab approved experiment E12-16-010: A Search for Hybrid Baryons in Hall B with CLAS12," (2016).

[22] J. Dudek et al., Eur.Phys.J. A48, 187 (2012), arXiv:1208.1244 [hep-ex],

[23] H. Al Ghoul et al. (GlueX), Phys.Rev. C95, 042201 (2017), arXiv:1701.08123 [nucl-ex]

[24] M. R. Shepherd (GlueX), in Proceedings, 10th Conference on the Intersections of Particle and Nuclear Physics (CIPANP 2009): San Diego, USA, May 26-31, 2009, Vol. 1182 (2009) pp. 816-819.

[25] H. Kamano, S. X. Nakamura, T. S. H. Lee, and T. Sato, Phys.Rev. C88, 035209 (2013), arXiv:1305.4351 [nuclth],

[26] A. V. Anisovich, R. Beck, E. Klempt, V. A. Nikonov, A. V. Sarantsev, and U. Thoma, Eur.Phys.J. A48, 15 (2012), arXiv:1112.4937 [hep-ph].

[27] A. V. Anisovich, R. Beck, E. Klempt, V. A. Nikonov, A. V. Sarantsev, and U. Thoma, Eur.Phys.J. A48, 88 (2012), arXiv:1205.2255 [nucl-th]

[28] C. Fernández-Ramírez, I. V. Danilkin, D. M. Manley, V. Mathieu, and A. P. Szczepaniak, Phys.Rev. D93, 034029 (2016), arXiv:1510.07065 [hep-ph]

[29] C. Fernández-Ramírez, I. V. Danilkin, V. Mathieu, and A. P. Szczepaniak, Phys.Rev. D93, 074015 (2016), arXiv:1512.03136 [hep-ph]

[30] D. Rönchen, M. Döring, F. Huang, H. Haberzettl, J. Haidenbauer, C. Hanhart, S. Krewald, U. G. Meißner, and K. Nakayama, Eur.Phys.J. A50, 101 (2014), [Erratum: Eur.Phys.J.A51,no.5,63(2015)], arXiv:1401.0634 [nucl-th]

[31] D. Rönchen, M. Döring, H. Haberzettl, J. Haidenbauer, U. G. Meißner, and K. Nakayama, Eur.Phys.J. A51, 70 (2015), arXiv:1504.01643 [nucl-th].

[32] W.-T. Chiang, S.-N. Yang, L. Tiator, and D. Drechsel, Nucl.Phys. A700, 429 (2002), arXiv:nucl-th/0110034 [nucl-th]

[33] R. L. Workman, M. W. Paris, W. J. Briscoe, and I. I. Strakovsky, Phys.Rev. C86, 015202 (2012),
arXiv:1202.0845 [hep-ph]

[34] W. J. Briscoe, A. E. Kudryavtsev, P. Pedroni, I. I. Strakovsky, V. E. Tarasov, and R. L. Workman, Phys.Rev. C86, 065207 (2012), arXiv:1209.0024 [nuclth]

[35] A. V. Anisovich et al., Eur.Phys.J. A52, 284 (2016), arXiv:1604.05704 [nucl-th]

[36] D. Ruić, M. Mai, and U.-G. Meißner, Phys.Lett. B704, 659 (2011), arXiv:1108.4825 [nucl-th]

[37] R. Dolen, D. Horn, and C. Schmid, Phys.Rev.Lett. 19, 402 (1967)

[38] P. D. B. Collins, An Introduction to Regge Theory and High-Energy Physics, Cambridge Monographs on Mathematical Physics (Cambridge Univ. Press, Cambridge, UK, 2009).

[39] W. Braunschweig, W. Erlewein, H. Frese, K. Luebelsmeyer, H. Meyer-Wachsmuth, D. Schmitz, A. Schultz Von Dratzig, and G. Wessels, Phys.Lett. B33, 236 (1970)

[40] R. L. Anderson, D. Gustavson, J. R. Johnson, I. Overman, D. Ritson, B. H. Wiik, and D. Worcester, Phys.Rev. D4, 1937 (1971)

[41] J. Dewire, B. Gittelman, R. Loe, E. C. Loh, D. J. Ritchie, and R. A. Lewis, Phys.Lett. B37, 326 (1971)

[42] G. F. Chew, M. L. Goldberger, F. E. Low, and Y. Nambu, Phys.Rev. 106, 1345 (1957)

[43] A. V. Anisovich, C. A. Baker, C. J. Batty, D. V. Bugg, L. Montanet, V. A. Nikonov, A. V. Sarantsev, V. V. Sarantsev, and B. S. Zou, Phys.Lett. B542, 8 (2002), arXiv:1109.5247 [hep-ex]

[44] A. V. Anisovich, C. A. Baker, C. J. Batty, D. V. Bugg, L. Montanet, V. A. Nikonov, A. V. Sarantsev, V. V. Sarantsev, and B. S. Zou, Phys.Lett. B542, 19 (2002) arXiv:1109.5817 [hep-ex]

[45] J. Mandula, J. Weyers, and G. Zweig, Ann. Rev. Nucl. Part. Sci. 20, 289 (1970).

[46] J. Nys, V. Mathieu, C. Fernández-Ramírez, A. N. Hiller Blin, A. Jackura, M. Mikhasenko, A. Pilloni, A. P. Szczepaniak, G. Fox, and J. Ryckebusch (JPAC), Phys.Rev. D95, 034014 (2017), arXiv:1611.04658 [hepph].

[47] V. Mathieu, G. Fox, and A. P. Szczepaniak, Phys.Rev. D92, 074013 (2015), arXiv:1505.02321 [hep-ph] 